\title{
Invited editors' preface
}

This special edition of Arabian Archaeology and Epigraphy is dedicated to Neolithic studies on the Arabian Peninsula. Contributions cover a wide range of topics, and were prepared by participants of an Exploratory Workshop of the European Science Foundation held in Lyon, France, in April 2012. We had great pleasure in organising this meeting entitled 'The Neolithic of Arabia: New Paradigms and Future Perspectives', and we were honoured to reunite so many esteemed and committed colleagues from Europe, America and Arabia. Attended by about thirty participants, the workshop dealt with the Arabian Neolithic, a period that represents one of the most momentous advancements in human prehistory. The Neolithic period coincided with the beginning of the Holocene and saw the advent of a food-producing economy, as opposed to an economy based on hunting and gathering in several areas of the world. During the discussions it appeared that the social

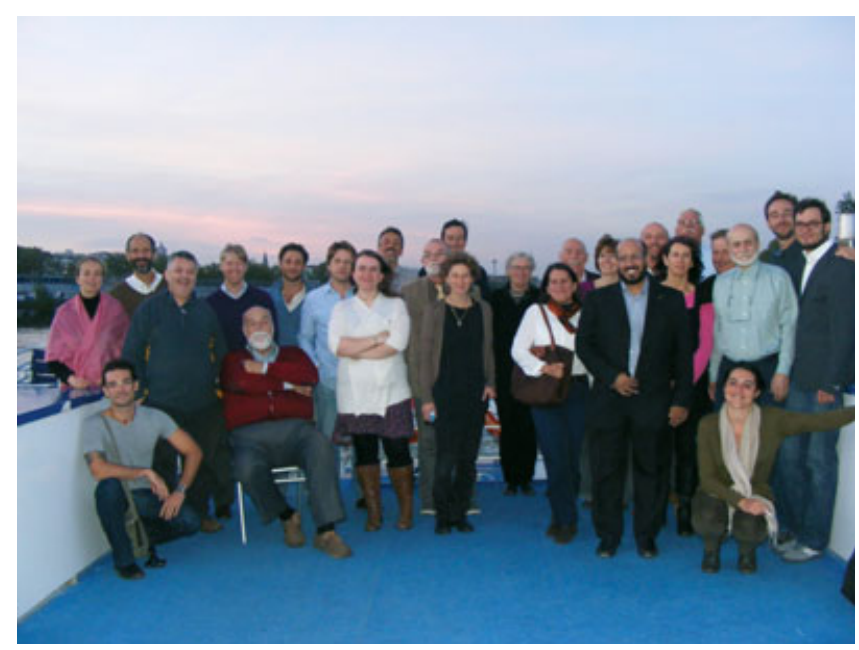

Fig. 1 .

Participants of the ESF Exploratory Workshop 'The Neolithic of Arabia: New Paradigms and Future Perspectives' held in Lyon (France), 24-27 April 2012, aboard a boat on the Rhône river: Margareta Tengberg, Yamandu Hilbert, Jean-François Berger, Mark Beech, Adrian Parker, Maurizio Tosi, Jeffrey Rose, Heiko Kallweit, Christine Kainert, Fabio Cavulli, Pléh Csaba (ESF Representative), Vincent Charpentier, Lamya Khalidi, Margarethe Uerpmann, Sophie Méry, Hans-Peter Uerpmann, Joy McCorriston, Abdullah Alsharekh, Richard Cuttler, Lucie Dingwall, Juris Zarins, Robert Carter, Francesco Fedele, Olivia Munoz, Rémy Crassard, Philipp Drechsler. impact of the Neolithic in Arabia was more visible than the purely economic one, as seen elsewhere in the world. Changes in exchange networks, as well as the dispersal of ideas, seem to have been more influential for the course of the Neolithic than just the emergence and development of a production economy.

Following many years of archaeological fieldwork, the Arabian Peninsula should no longer be considered as a dead end for more prosperous civilisations from the north, but as a region within which distinctive developments ultimately led to the establishment of independent civilisations. Two research schools have dominated the scientific debate on the origin and development of the Neolithic on the Arabian Peninsula, one favouring the dominance of indigenous developments, the other emphasising influence from abroad. Dissatisfied with an ongoing and stagnating discourse, we had a vision that bringing together all the researchers from different schools and institutions would both stimulate the development of new paradigms and contribute to the establishment of a comprehensive and coherent image of the Neolithic of the Arabian Peninsula.

The results of discussions, if not strong debates, are presented in the following pages. Papers are geographically organised to allow the reader more easily to link different regions and sites, as well as concepts and theories. Main topics cover definitions of concepts that are sometimes very peculiar to Arabia, as well as chronology and associated material culture that is mostly represented by the lithic industries. Actual hypotheses on the development of the Neolithic in Arabia are also largely debated and based on different approaches: palaeoenvironments, climatic changes, typology and technology and general aspects of population behaviours and settlement patterns.

This workshop marks a first milestone in the Arabian Neolithic research community, allowing the informal foundation of a scientific group of archaeologists and other scientists, the acronym ISNAP (International Symposium on the Neolithic of the Arabian Peninsula) having been chosen to represent the group. We propose organising a meeting every two years, the next one being in preparation for 
2014/2015. Future meetings will allow a larger group of delegates than at Lyon, and will also embrace wider perspectives, such as neighbouring regions and periods (e.g. Palaeolithic and/or Bronze Age).

This AAE Special Issue has been possible thanks to Prof. Dan T. Potts who accepted us as invited editors. We wish to thank the Wiley editorial team for their help. The Exploratory Workshop in Lyon was mainly and generously financed by a European Science Foundation grant (EW11-034), by the CNRS Institut National des Sciences Humaines et Sociales and by the Association des Amis de la Maison de l'Orient. We would like to offer them our profound thanks. Finally, we wish to thank the many people in Lyon who got involved in the planning and generously gave of their time: Gwénaëlle Péquay, Jwana Chahoud, Emmanuelle Vila, Virginie Huyard, Marie Le
Mière, Olivier Aurenche, Christine Chataigner and Frédéric Abbès, who all are members of the CNRS team Archéorient, UMR 5133. Staff from the Maison de l'Orient et de la Méditerranée also generously assisted, especially Anne Schmitt, Nathalie Donjon and Claudine Maréchal.

\author{
Rémy Crassard ${ }^{1}$ and Philipp Drechsler ${ }^{2}$ \\ ${ }^{1}$ Centre National de la Recherche Scientifique CNRS - \\ UMR 5133, Maison de l'Orient et de la Méditerranée, \\ Lyon, France \\ ${ }^{2}$ Universität Tübingen Institut für Ur- und Frühgeschichte, \\ Tübingen, Germany
}

